\title{
Experimental Mechanical Jaundice: Morphological Aspects of Proliferation, the Possibility of Transformation and Involution of Epithelial Cells in the Bile Ducts
}

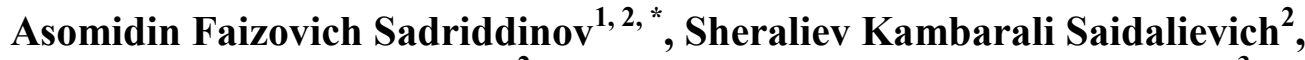 \\ Hidirova Gulnoza Azodovna ${ }^{2}$, Muborak Asomidinovna Sadriddinova ${ }^{3}$, \\ Elena Nikolaevna Guzacheva ${ }^{4}$ \\ ${ }^{1}$ Department of Histology and Pathological Physiology, Tashkent Pediatric Medical Institute, Tashkent, Uzbekistan \\ ${ }^{2}$ Department of Anatomy and Pathological Anatomy, Tashkent Pediatric Medical Institute, Tashkent, Uzbekistan \\ ${ }^{3}$ Department of Emergency Pediatrics, Tashkent Pediatric Medical Institute, Tashkent, Uzbekistan \\ 4"Xayat" - Private Clinic, Tashkent, Uzbekistan
}

Email address:

asom_sad23@mail.ru (A. F. Sadriddinov)

${ }^{*}$ Corresponding author

\section{To cite this article:}

Asomidin Faizovich Sadriddinov, Sheraliev Kambarali Saidalievich, Hidirova Gulnoza Azodovna, Muborak Asomidinovna Sadriddinova, Elena Nikolaevna Guzacheva. Experimental Mechanical Jaundice: Morphological Aspects of Proliferation, the Possibility of Transformation and Involution of Epithelial Cells in the Bile Ducts. International Journal of Clinical and Experimental Medical Sciences.

Vol. 7, No. 4, 2021, pp. 98-102. doi: 10.11648/j.ijcems.20210704.15

Received: June 8, 2021; Accepted: July 8, 2021; Published: July 24, 2021

\begin{abstract}
On an experimental model of obstructive jaundice in experimental rats, the cytological aspects of proliferation and the possibility of transformation of cholangiol cells into hepatocytes, as well as the cellular mechanisms of involution of the bile ducts, were investigated. The material was the liver of sexually mature rats $(n=38)$ with 10,20 , and 25 days of cholestasis, in which the duct was recanalized during these periods and sacrificed on the 5th, 10th, 15th, 30th and 90th days after the second operation. Pieces of liver tissue were processed by conventional histological and electron microscopic methods. Ligation of the bile duct promotes rapid proliferation of the bile ducts, which correlates with the duration of cholestasis. In the early stages of the experiment (cholestasis for 10 days), the bile ducts occupy the peripheral sections of the lobules, by the 20th day, cholestasis penetrates deeply into the lobule, dismembering it into fragments, and by the 25th day, a picture of biliary cirrhosis develops. It was found that the proliferation of the bile ducts occurs due to the duct cells themselves, the transformation of cholangiol cells into "oval" cells or hepatocytes was not found. The transformation criteria were the shape, intracellular structures, and the state of the boundary plate and the basement membrane of the ducts. Consequently, bile duct cells (cholangioli) and hepatocytes, diverse in their function, showed absolute genetic heterogeneity. After the restoration of the outflow of bile, non-functioning ducts undergo destruction and decay. It has been shown that hepatic macrophages play an active role in cleansing the liver parenchyma from the products of cellular decay of the bile ducts, dead liver cells, as well as excess fibrillar structures. Activated Kupffer cells, due to the content of a large number of heterolysosomes, provide degradation, lysis and utilization of cellular and extracellular structures.
\end{abstract}

Keywords: Obstructive Jaundice, Liver, Proliferation, Transformation and Involution of Cells

\section{Introduction}

Numerous studies determined that some diseases and experimental liver lesions were accompanied by hyperplasia of bile ducts [1-3]. The slight vulnerability of bile ducts, the famous scientist Ashoff (the famous scientist) called them the "Achilles heel" of the liver. Recently, the data appeared that hepatocytes can be formed on the account of cholangiol cells or oval-stem cells $[4,5]$. It was determined that ligation of common bile duct was accompanied by the rapid proliferation 
of bile ducts [6-8]. Thus, on given model it can be investigated the transformation of cholangiol cells, and, simultaneously to the fate of hyperplastic bile ducts after restoration of bile outflow. And, coming from it, the aim of the work was to study the relationship of bile duct cells with hepatocytes during cholestasis on the model of experimental mechanical obstructive jaundice, as well as to observe the fate of occurring tubular structures after recanalization of bile duct.

\section{Materials and Methods of Study}

The experimental model of mechanical obstructive jaundice was created by ligation of common bile duct at experimental white rats $(\mathrm{n}=38)$. Then, in 10, 20 and 25 days of cholestasis, the recanalization of bile ducts was performed, the experimental animals of each period were killed in 5, 10, 15.30 and 90 days after restoration of bile outflow. The keeping, killing of experimental animals were carried with the following ethical standards norms set from the "Rules of work with experimental animals."

The pieces of liver tissue for histological studies, after fixation in $12 \%$ solution of neutral formalin and standard processing, were poured in mixture of paraffin and wax. Sections for histological studies were stained with hematoxylin - eosin. Some pieces were processed by general methods of electron microscopic studies.

\section{Research Results}

After ligation of common bile duct at early stages of experiment (10th day), the proliferated bile ducts occupy the portal fields, they partially penetrate into peripheral parts of lobules and contact close with hepatocytes. As jaundice increases (20th day), the tubular structures penetrate deeply into the lobule, dismembering hepatic plates into separate islets or fragments. By the 25th day of cholestasis, the biliary cirrhosis was formed in the liver; in isolated islets, along with less altered hepatocytes, the atrophied and necrotic cells were found (Figure 1). Therefore, on the height of cholestasis, the liver parenchyma was completely disorganized and the newly formed bile ducts were dilated, the basal membrane was thickened, the epithelial cells were flattened themselves and were without edges, but the intracellular organelles were less changed. In comparative study ultrastructure of hepatocytes and cholangiols, it was found out that they differ significantly from each other in shape and intracellular structure. The epithelial cells had cubic shape and poor in subcellular organelles, while hepatocytes were large, polygonal or oval in shape and rich in intracellular organelles. The direct approach of epithelium of bile ducts and small cells cholangiols, being also called oval cells with hepatocytes, create ideal conditions for clarifying the mutual transformation of cells. To elucidate the process of cholangiols transformation into hepatocytes, in addition to the shape and development of subcellular organelles, the important identifying feature was the state of basal membrane present in the duct cells and its absence in hepatocytes. Our electron microscopic studies showed that all overgrown duct epithelial cells, as a rule, were localized on basal membrane, and we did not notice the ruptures of membrane and the movement of cholangiol cells towards hepatocytes. Moreover, the appearances of intermediate, transitional or oval cells were also not identified. In addition, during development of experimental model of mechanical obstructive jaundice in isolated cases, we discovered an unusual phenomenon (casuistic cases), when the bile ducts proliferated in unusual way. The proliferated bile ducts were spread exclusively along the portal tracts, forming the wide strip between adjacent lobules. The morphological analysis of this phenomenon showed that the proliferated bile ducts did not violate the border plates and introduction of epithelial cells into the lobule were not observed. The increase number of bile ducts was associated with proliferation of epithelial cells themselves, as evidenced by detection of mitotic figures in these cells. Therefore, at cholestasis, only epithelium of bile ducts is undergone to active proliferation, the appearance of oval or intermediate cells resembling cholangiol or hepatocyte cells were not notied.

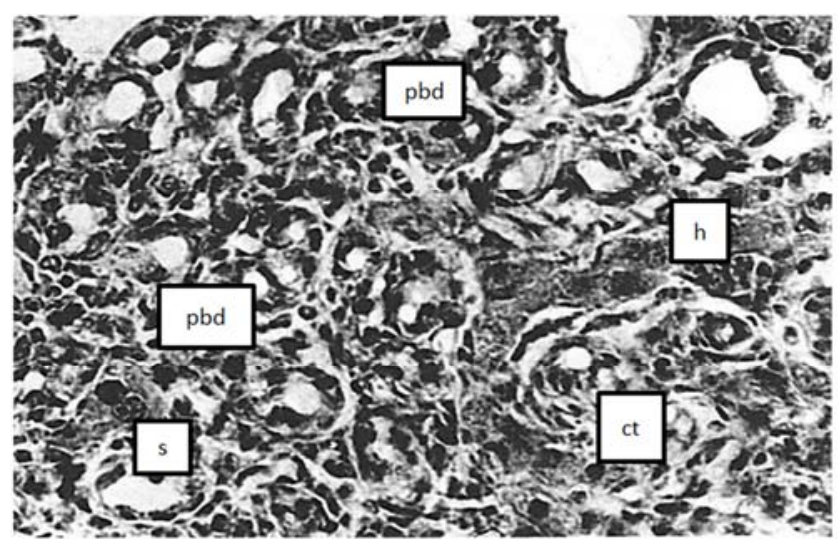

Figure 1. Rat liver on the 5th day of recovery of 25 daily cholestasis. The liver parenchyma is replaced by numerous proliferated tubular structures, between which the isolated hepatocytes are revealed. Designation here in after: H-hepatocytes; PBD-proliferated bile ducts; S-sinusoids; CTconnective tissue. Coloring with hematoxylin and eosin. Mg. 200.

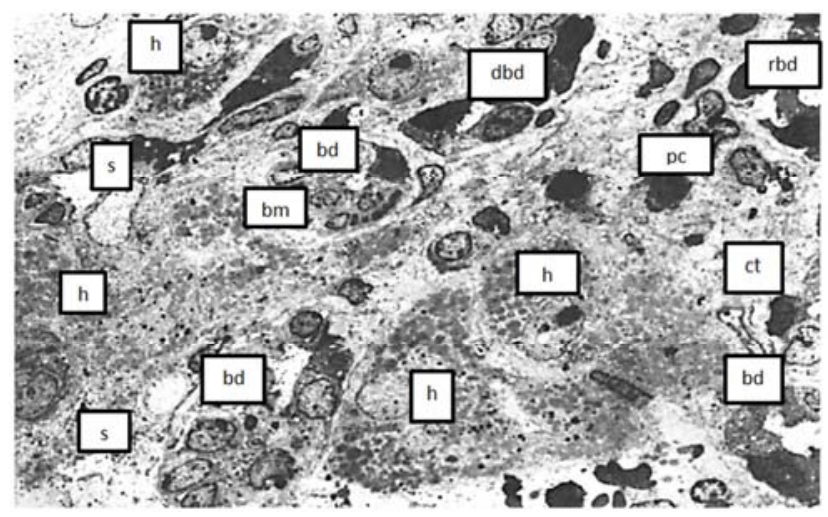

Figure 2. Rat liver on the 5th day of recovery of 20 daily cholestasis. Divided parenchyma by proliferated bile ducts. In the islets the isolated hepatocytes have the same structure. But the proliferated bile ducts show a heterogeneity, some ducts are light others are dark (explanation in the text). S-sinusoids; RBD-reducing bile ducts; PC-pycnosis of the cell; BM-basal membrane; BD-bile duct; Mg. 6000. 
After outflow recovery in 10, 20 and 25-day of cholestasis, the lobules that have penetrated into interior, and, most of peripheral bile ducts on the 5 th day of recovery were turned off from the circulation system, indicating their secondary importance. The functioning ducts were characterized by wide lumen, the epithelial cells were well preserved, the shape of cells was unchanged, and the cytoplasm contained the sufficient number of intracellular organelles. Meanwhile, reducing tubular structures were represented by prolonged tubular or rounded formations, being found both on periphery and inside the lobules, in the inter-hepatocyte or per sinusoidal spaces (Figure 2). Such decaying structures were usually delimited from liver cells with thickened basal membrane and connective tissue elements. These destructive ducts have the narrow lumen, and, mainly, the structure of lining cells was dramatically changed. The cells were characterized with the dense matrix, the intracellular organelles were not identified, and, some of cells were in state of dissociation or separation from the duct wall (Figure 2 ). The rejected cells, along with pyknosis of cytoplasm and nucleus, sometimes had vacuoles and myelin figures. The involution of duct, apparently, began with destruction of intercellular connections, after which the short outgrowths appeared on the surface of cells, the basal membrane disappeared, and the remains of cell became more shrink and turned out in inter-hepatocyte spaces. If stellate macrophages were absent near such foci of destruction, the cellular remains stay for a long time, as it was evidenced of detection such structures on the 90th day of postoperative period (Figure 3). Usually, Kupffer cells were evenly scattered in the liver, but their number increased, especially in foci of cell destruction or along the sinusoids, where dead hepatocytes, were undergone to destruction as well as stromal elements, also undergone to decay. The activated macrophages had prolonged shape, large size, and contained a large number of hetero-lysosomes in cytoplasm. The important sign of activation was also the appearance of numerous filo podia and microvilli on the surface of macrophages. Capture and enclosing the decaying structures by several macrophages, the cytoplasm of which were filled with the large number of phagocytized parts of cells, vacuoles, vesicles, residual bodies of various shapes and densities were shown (Figure 4), in some macrophages it was observed the rupture of membranes and release of lysosomes into the intercellular gap. Obviously, under the action of lysosomal enzymes, the nearby cell was destroyed first, and then the remaining cells of the duct are destroyed too. Some macrophages are able to catch large shriveled cell remains, which were completely cleaved by lysosomes to smaller microstructures, and then, like other phagocytic particles, were utilized by the cell. Obviously, non-split residual bodies were released into the intercellular environment or carried away by the blood stream (Figure 5). Cleaning liver parenchyma from excess structures and products of cellular degradation became favors for restoration of hepatocytes. Moreover, the reparative regeneration of liver cells manifested itself in the form of hyperplasia of the cytoplasmic reticulum, expansion of its tubules, the increase in the number of mitochondria, lysosomes, hypertrophy of lamellar complex and the formation of long microvilli on their sinusoidal surface.

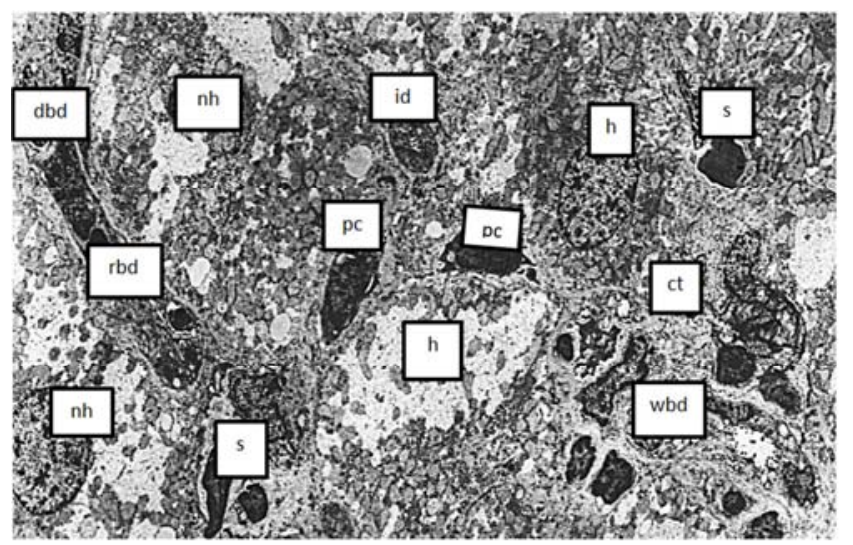

Figure 3. Rat liver on the 15th day of recovery of 20 daily cholestasis. Some improvement of morphological picture hepatic plates and sinusoids. The reduction of bile ducts continues, some of them are located between hepatocytes and sinusoid capillaries others are in state of disorganization and destruction. NH-hepatocyte nucleus; WBD-walled up bile ducts. IDimmured duct; BD- bile duct; DWP-destruction of the bile duct; RBDreduction of the bile duct. $M g$. 6000 .

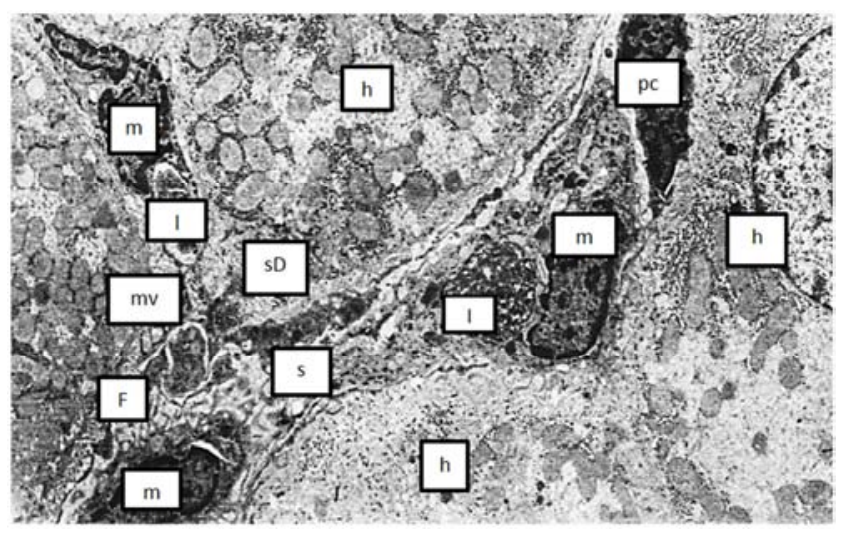

Figure 4. Rat liver on the 30th day of recovery of 20 daily cholestasis. Full restoration of intrahepatic structures for hepatic cells. The sinusoid lumens are occupied with activated macrophages the cytoplasm of those contains numerous phagolysosomes with caught remains of epithelial cells ducts and elements of connective tissue. F-filopodia of macrophage; L-lysosomes; $s D$ space of Disse; $M V$ - microvilli of hepatocytes. 7600.

Thus, after recanalization in 10 days of daily cholestasis, the proliferated bile ducts were undergone to involution and disappeared by the 15 th day after recanalization. At 20 and 25 day of cholestasis, the intralobular ducts were undergone to involution by the 30th day after operating period at participation of hepatic macrophages, but some immured remains in interhepatocytic or peri- sinusoidal spaces are kept for a long time. Therefore, involution of bile ducts occurred much more slowly than the restoration of parenchymal structures, the main element of which was hepatic cell.

\section{Discussion of Taken Results}

The taken studies showed that at experimental rats, unlike other animals, the ligation of common bile duct was 
accompanied by rapid proliferation of bile ducts, probably due to the absence of gall bladder there. Besides, the process of duct proliferation correlated with duration of cholestasis, so, the biliary cirrhosis of the developed in animals at relatively short period ( 25 days). At the initial stages of jaundice (10 days), the bile ducts were wedged into peripheral parts of lobules, and at 20 and 25-day cholestasis, they completely disorganized the parenchyma of organ. Moreover, it created a real opportunity to study mechanism of transformation of cholangiol cells into hepatocytes. Ultra structural analysis of cholangiols and hepatocytes in obstructive mechanical jaundice made it possible to establish occurrence of "oval" (in the literature, this term presumably denoted cholangiol cells), $[9,10]$, or intermediate cells were not identified. In addition to the intracellular structure of cells, the criteria for transformation were their topography, as well as the state of basal membrane of epithelium. The additional arguments were the unusual or atypical cases of cells proliferation, rarely occurring during obturation. The critical analysis of results obtained allowed proving that cholangiol cells do not transform into hepatocytes, moreover, the cells with oval shape did not appear. It was shown by some researchers [11-13]. Therefore, bile duct cells of various functions (cholangioli) and hepatocytes had absolute genetic heterogeneity.

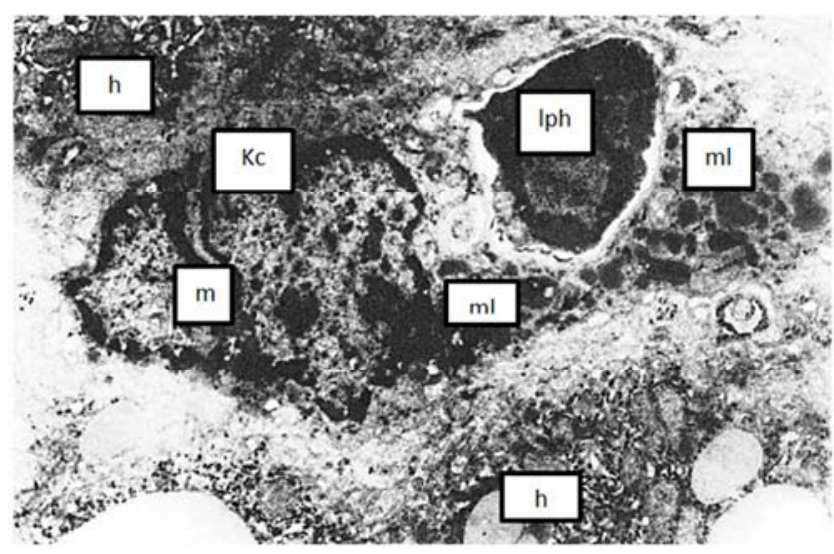

Figure 5. Rat liver on the 30th day of recovery of 25 daily cholestasis. Phagocytosis of large remain of dead cells, it may be epithelium duct being killed by macrophage, in cytoplasm of which are also a large number of lysosomes, participating in degradation, lysis and utilization of cellular and extracellular structures. LPH-large phagolysosome; ML-multiple lysosomes Mg 10000.

Observations of recovery dynamics processes after recanalization made it possible to establish that, at first, the secondary ducts were excluded from the circulation system, and then these ducts were undergone to reduction and disorganization. The involution of reducing bile ducts occurred with direct participation of liver macrophages. The activated cells moved to destructively altered structures, probably, firstly, they secrete lysosomal enzymes, under the action of which the cells of the duct wall break down and disintegrated. Then, by phagocytosis, the disintegrated parts of cells were processed by lysosomes and utilized. It should be noted that macrophages act in a similar way towards dead hepatocytes, fibrous structures and fibrocytes [14, 15]. Apparently, only stellate macrophages of liver were able to capture and process the reducing ducts with lysosomes; the participation of other cells, including hepatocytes, was not noted. It was evidenced by detection of shrunken ducts or cell remains in inter-hepatocytes or per sinusoidal spaces in the long term (90 days) of observation. Thus, the taken studies showed that at the period of cholestasis, the rapid proliferation of bile ducts was observed in the liver, being associated with the mitotic division of epithelial cells themselves. At the same time, the appearance of oval or intermediate cells being capable to transform into hepatocytes were not marked. When the outflow of bile was restored, the proliferated bile ducts were firstly turned off from circulation system, after which they were undergone to reverse development decay and utilization. In such process, the activated liver macrophages, containing numerous lysosomes of various sizes and densities play the important role.

\section{Conclusion}

1) Blockage of the bile duct is accompanied by rapid proliferation of the bile ducts. The formation of new ducts is carried out due to the division of the epithelial cells themselves.

2) During the proliferation of epithelial cells, their transformation into hepatocytes or vice versa, as well as the appearance of "oval" or intermediate cells, we did not find in this study.

3) Reverse development, disorganization, disintegration of reducing cells of the bile ducts are associated with the activation of liver macrophages - Kupffer cells.

\section{References}

[1] Aruin, L. I. Apoptosis and liver pathology. RJGH and C. 1998. No. 2. - P. 6-11.

[2] Mayansky, D. N. Immunological properties of sinusoidal liver cells. Advances in modern biology. - 1992.-T. 112, No. 1. - P. $100-114$.

[3] Petrosyan T. R. Cells involved in liver regeneration: stem cells, oval cells, ITO cells, hybrid hepatocytes. stem cells in the genesis of HCC and the treatment of cirrhosis. Synergy of Sciences. - 2017. - T. 1, No. 17. - P. 927-933.

[4] Factor V. M., Radaeva S. A. Stem reserve of the liver. Ontogenesis 1991; 22 (2): 181-9.

[5] Crosby H. A., Kelly D. A., Strain A. J. Human hepatic stem-like cells isolated using c-kit or CD34 can differentiate into biliary epithelium. Gastroenterology 2001; 120 (2): 863-70.

[6] Chen Y.-K., Zhao X.-X., Li J.-G et al. Ductular proliferation in liver tissues with severe chronic hepatitis B: An immunohistochemical study. World J. Gastroenterol. 2006; 12 (9): 1443-6. 
[7] Sadriddinov, A. F., Abdullaeva N. E., Sadriddinova M. A., Fakhriev J. A., Shoyunusov S. I. The classic hepatic lobule is in three dimensions: organization, cytoacrchitecture and hem circulatory mainstream of simple hepatic lobule for rat and human. European Science Review, Austria, and Vienna. 2017, № 5-6, p. 36-41.

[8] Kashaeva M. D., Proshin A. V., Afanasyev A. N., Gavrilova K. V., Golushko A. V. Liver morphology in obstructive jaundice. Bulletin of Novgorod State University, 2018; No. 6 (112). P. $8-11$.

[9] Hsia C. C. Evarts R. P., Nakatsukasa H. et al. Occurrence of oval cell in hepatitis B virus associated human hepatocarcinogenesis. Hepatology 1992; 67: 427-33.

[10] Parent R., Marion M. J., Furio L. et al. Origin and characterization of a human bipotent liver progenitor cell line. Gastroenterology 2004; 126 (4): 1147-56.

[11] He Z. P., Nan W. G., Nang Y. F. et al. Activation, isolation, identification and in vitro proliferation of oval cells from adult rat liver. Cell Prolife. 2004; 37 (2): 177-87.
[12] Kiyasov A. P., Gumerova A. A., Titova M. A. Oval cells putative liver stem cells or hepatoblasts? Cell transplantology and tissue engineering. 2006, No. 2 (4), - C, 55-58.

[13] Engelhardt N. V., Factor V. M., Medvinsky A. L. et al. Common antigen of oval and biliary epithelial cells (A6) is a differentiation marker of epithelial and erythroid cell lineages in early development of the mouse. Differentiation 1993; 55: P. 19-26.

[14] Klein I., Cornejo J. C., Polakos N. K., John B., Wuensch S. A., Topham D. J. Pierce R. N., Crispe I. N. Kupffer cell heterogeneity: functional properties of bone marrow derived and sessile hepatic macrophages. Blood. - 2007. -Vol. 110 (12). - P. 4077-4085.

[15] Shevaldin A. G. Resident macrophages of the liver and the absolute number of circulating monocytes in chronic viral hepatitis C. HIV infection and immunosuppression. 2014; 6 (3): 24-31. https//doi.org/10.22328/2077-9828-2014-6-3-2431 . 